\title{
Evaluation of myocardial viability following ischemic and reperfusion injury using phosphorus 31 nuclear magnetic resonance spectroscopy in vivo
}

\begin{abstract}
Recovery of myocardial high-energy phosphate (HEP) metabolism after coronary occlusion and reperfusion may vary with ischemic duration and may provide information about the extent of tissue viability. To evaluate the differences between varying durations of ischemia and to attempt to identify metabolic indexes of salvaged viable tissue, intact New Zealand white rabbits underwent either 30 (group $1 ; n=8$ ) or 60 (group 2; $n=8$ ) minutes of coronary occlusion followed by reperfusion. HEP metabolism was evaluated with cardiac gated phosphorus $31\left({ }^{31} \mathrm{P}\right)$ nuclear magnetic resonance (NMR) spectroscopy with a $2.0 \mathrm{~T}$ spectrometer. While similar HEP changes were observed during ischemia in both groups, differences in HEP recovery between groups were seen following reperfusion. Group 1 animals demonstrated a gradual decrease in inorganic phosphates $(\mathrm{Pi})(p<0.05$ versus group 2$)$, an immediate recovery of phosphocreatine $(\mathrm{PCr})(p=n s$ versus baseline), and a gradual increase of adenosine triphosphate (ATP) to pre-ischemic levels. Group 2 animals had elevated levels of $\mathrm{Pi}(p<0.05$ versus baseline; $p<0.05$ versus group 1), slow recovery of PCr, and continued reduction of ATP ( $p<0.05$ versus baseline; $p<0.05$ versus group 1). Group 1 rabbits had a greater extent of viable myocardium than group $2(77.1 \pm 9.7 \%$ of risk area versus $39.4 \pm 9.4 \% ; p<0.001)$. Significant correlations were found between $\mathrm{PCr}$ and $\mathrm{Pi}$ reperfusion values and myocardial viability $(r=0.59, p<0.05 ; r=0.73$, $p<0.01$, respectively). Thus differences between metabolic recovery after different durations of ischemla Indicate the existence of graded metabolic recovery dependent upon the severity of the ischemic insult, and the correlations observed suggest that metabolic data may indicate tissue viability. HEP metabolic information available through the non-tissue destructive method of ${ }^{31} \mathrm{P}$ NMR spectroscopy may be useful in the evaluation of postischemic myocardial viability. (AM HEART J 1990; 120:31.)
\end{abstract}

Lynette C. Wroblewski, BS, Alex M. Aisen, MD, Scott D. Swanson, PhD, and Andrew J. Buda, MD. Ann Arbor, Mich.

As the methods and frequency of clinical coronary reperfusion following acute myocardial infarction increase, there exists an increasing need for techniques that can evaluate the viability and potential recovery of salvaged cardiac tissue. While aggressive interventions such as coronary thrombolysis, percutaneous angioplasty, and coronary artery bypass sur-

From the Cardiology Division. Department of Internal Medicine, and Department of Radiology, University of Michigan Medical School.

This work was supported in part by a grant-in-aid from the American Heart Association of Michigan, and by Grant R01 HL-34691 from the National Heart, Lung, and Blood Institute, National Institutes of Health, Bethesda, Md. Ms. Wroblewski was supported by a Student Research Fellowship from the American Heart Association of Michigan, Lathrup Village, Mich.

Received for publication Jan. 5, 1990; accepted Feb. 15, 1990.

Reprint requests: Andrew J. Buda, MD, Cardiology Division, TC 3910 Box 0366, University of Michigan Medical Center, 1500 East Medical Center Dr., Ann Arbor, MI 48109-0366.

4/1/20523 gery may be implemented to revascularize ischemic myocardium, the methods used to characterize tissue salvaged by these interventions have crucial limitations. Echocardiography and thallium scintigraphy provide functional and perfusion information that does not accurately identify viable tissue due to the myocardial stunning phenomenon ${ }^{1}$ or because of complexities of thallium uptake. ${ }^{2}$ Positron emission tomography (PET imaging) is used to directly evaluate metabolic activity via substrate uptake, but this method may have limitations due to delayed metabolic recovery following an acute ischemic insult. ${ }^{3}$ Therefore the development of alternative methods with which to assess myocardial viability is desirable.

The noninvasive, non-tissue destructive method of phosphorus 31 nuclear magnetic resonance spectroscopy ( $\left.{ }^{31} \mathrm{P} \mathrm{NMR}\right)$ is a new technique that offers potential for assessing metabolic injury and recovery. 
${ }^{31} \mathrm{P}$ NMR directly evaluates the levels of high-energy phosphates (HEP) present in myocardium, a direct indication of the amount of energy available for myocardial viability and contractile function. Depletion of adenosine triphosphate (ATP) and other high-energy phosphate molecules such as phosphocreatine (PCr) may account for and/or contribute to the dysfunction of insulted myocardium and the disruption of myocardial cell integrity. ${ }^{4}$ Therefore the technique of ${ }^{31} \mathrm{P}$ NMR spectroscopy to evaluate HEP metabolism may be useful in the assessment of reperfused myocardium.

Typical changes in HEP metabolism during periods of coronary artery occlusion and reperfusion using the technique of ${ }^{31} \mathrm{P}$ NMR have been reported previously by many investigators. ${ }^{5-8}$ The experimental models have provided information about energy metabolism following myocardial injury. More recently, it has been demonstrated that it is possible to perform image-selected in vivo spectroscopy on the human heart, ${ }^{9}$ and it has been suggested that this noninvasive technique may ultimately be useful in the clinical diagnosis and evaluation of cardiac disorders. ${ }^{10-12}$ Because of its noninvasive capabilities along with its ability to directly evaluate HEP stores, ${ }^{31} \mathrm{P}$ NMR spectroscopy has the potential to accurately characterize tissue viability. ${ }^{13}$ To further investigate the role of ${ }^{31} \mathrm{P}$ NMR spectroscopy to evaluate myocardial viability in a clinically relevant model of coronary occlusion and reperfusion, we designed a study that examined the differences in HEP metabolic recovery using ${ }^{31} \mathrm{P}$ NMR spectroscopy following different grades of severity of myocardial injury.

\section{METHODS}

Animal model. Each of the following experimental procedures is in accordance with the "Guiding Principles in the Use and Care of Animals" approved by the Council of the American Physiological Society as well as with state and federal laws. Sixteen healthy New Zealand rabbits weighing 3.3 to $4.0 \mathrm{~kg}$ each were anesthetized with intramuscular injections of xylazine $(10 \mathrm{mg} / \mathrm{kg})$ and then ketamine $(40 \mathrm{mg} / \mathrm{kg})$. An internal jugular vein was isolated and cannulated for the administration of drugs and fluids. Rabbits were maintained under anesthesia by $0.1 \mathrm{ml}$ boluses of pentobarbital sodium as needed throughout the protocol. Animals were intubated via tracheostomy and were placed on a small animal Harvard respirator (Harvard Apparatus Co., S. Natick, Mass.) with supplemental oxygen. 'The apparatus and validation of adequate oxygen delivery are described in detail elsewhere. ${ }^{5} \mathrm{~A}$ left thoracotomy was performed through the fifth intercostal space, and the pericardium was gently excised, exposing the lateral surface of the left ventricle. A reversible snare occluder was created by placing a $6-0$ suture around the marginal branch of the subepicardial left circumflex coronary artery slightly distal to the atrioventricular groove and threading a stronger extension of this suture through a small plastic tube. Distal to the suture placement, the radiofrequency coil, which is described below in detail, was secured onto the myocardium that was to be rendered ischemic. The reliability of this occlusion model has been detailed previously. ${ }^{5}$ Electrocardiographic (ECG) leads, to allow cardiac gated spectral acquisitions, were stitched into the muscle of the animals' limbs with stainless steel sutures. Animals were surrounded by a heating pad of a water bath maintained at $37^{\circ} \mathrm{C}$ and were wrapped in insulated covering. The rabbits were then carried into the NMR unit and placed in the $22 \mathrm{~cm}$ bore of the magnet.

Nuclear magnetic resonance spectroscopy. A $2.0 \mathrm{~T}$ General Electric/Nicolet CSI-II nuclear magnetic resonance spectrometer (GE Medical Systems, Milwaukee, Wisc.) was used to collect spectral data. The copper wire four-turn radiofrequency coil had a diameter of $13 \mathrm{~mm}$ and an axial length of $6 \mathrm{~mm}$ to assure near optimum circuit $Q$ and concentration of the $B_{1}$ field along the coil's axis. This arrangement was able to maximize the signal received from the myocardial area of interest while minimizing any contamination by nonischemic tissue. Capacitance losses were reduced using a balanced tuning circuit ${ }^{14}$ whose unbalanced side was grounded within the magnetic bore. The silicone rubber that surrounded the coil and protected it from corrosion produced a short $\mathrm{T}_{2}$ (relaxation time) proton resonance that did not interfere with the proton water signal used in shimming. The maximum sensitivity of the coil was described at its surface through phamtom studies, and it was found to be $80 \%$ of the signal received within 6 $\mathrm{mm}$ of axial distance.

Once placed inside the magnet and aligned with the maximum intensity of the magnetic field, the animal was not moved. The coil was tuned to $34.62 \mathrm{MHz}$, the resonating frequency for phosphorus at this field strength. Due to the relative abundance of water protons within the biologic system, shimming was performed on the proton signal with a pulse width of $30 \mu \mathrm{s}$ in order to maximize field homogeneity. Water line widths of 0.3 to $0.5 \mathrm{ppm}$ were obtained. ${ }^{31} \mathrm{P}$ spectra were gated via $\mathrm{ECG}$ signals received at precisely the same point of the cardiac cycle to reduce unavoidable motion-related line broadening. After the collection of 256 transient averages, the time domain data was multiplied by an exponential function with $10 \mathrm{~Hz}$ line broadening and was Fourier transformed to yield the intensity versus frequency plot, i.e., the phosphorus spectrum.

For each spectrum, the areas under the five distinguishable phosphate peaks (inorganic phosphate [Pi], $\mathrm{PCr}$, alpha, beta, and gamma ATP) were calculated using a Lorentzian curve-fitting software program. Levels of ATP are represented by the $\beta$-ATP peak, since it is the most uncontaminated ATP resonance. Results are presented as relative percent areas of the total area of the five phosphate peaks. Since these areas will sum to $100 \%$, their values will mathematically correlate.

Experimental protocol. After gathering baseline spectra, the coronary artery was ligated and the accumulation 
of the occlusion data was initiated. Because each spectrum required $10 \pm 1$ minutes to be completed, animals were randomly assigned to either three $(n=8)$ or six $(n=8)$ consecutive collections, which resulted in either $30 \pm 3$ or $60 \pm 6$ minutes of ischemia, respectively. Following the appropriate duration of occlusion, the ligature was removed to allow reperfusion of the coronary artery. Three subsequent spectra were collected over a total of $30 \pm 3$ minutes. The rabbit was then removed from the bore of the magnet and was left undisturbed to allow a total reperfusion period of 3.0 to 3.5 hours.

A cannula was then placed into the left atrium and the artery was reoccluded. Approximately $5 \mathrm{ml}$ of Trypan blue stain was flushed through the left atrium to delineate the in vivo risk zone, while 5 to $10 \mathrm{ml}$ of potassium chloride were simultaneously introduced into the jugular vein to arrest cardiac contraction. Hearts were excised, and the left ventricle was isolated, frozen, and sliced into 8 to 15 segments. Slices were then weighed and soaked in a solution of triphenyltetrazolium chloride (TTC) for 15 minutes. ${ }^{15}$ Following overnight compression and fixation in formalin, heart slices, risk areas, and infarct regions were traced using an opaque projector that enlarged the small slices and allowed for improved differentiation between stained areas. Myocardium within the risk region (defined by Tripan blue) that was not infarcted was considered viable myocardium. The areas were quantified by planimetry and, with the respective weights, percentages of risk area, infarct area, and viable area were calculated.

Statistical analysis. The data generated through the GE/Nicolet Lorentzian software and the planimetry analysis were expressed as mean \pm SEM. Differences both between the experimental groups and among the various time points were identified through one-way analysis of variance (ANOVA) and then unpaired, two-tailed Student's $t$ tests were performed. Correlations between viability and HEP levels were calculated by linear regression analysis.

\section{RESULTS}

Hemodynamics. Heart rates of the animals were relatively constant over the time course of the protocol, with no significant difference calculated at each point relative to baseline. Also, no differences were seen between groups of animals.

NMR data. Temporal changes in the levels of HEP are represented by the NMR spectral stacked plots in Figs. 1 and 2. Fig. 1 is a typical plot of an animal in group 1 that was reperfused after 30 minutes of ischemia. In Fig. 2, a representation of group 2 animals, those reperfused after 60 minutes of ischemia are shown. Relative percent areas of the peaks corresponding to $\mathrm{Pi}, \mathrm{PCr}$, and $\mathrm{ATP}$ are summarized in Table I.

Marked changes in high-energy phosphates were observed immediately after the initial ischemic insult. In both groups of animals, levels of $\mathrm{Pi}$ increased rapidly from baseline levels and remained consis- tently elevated over time ( $p<0.05$ versus baseline), as shown in Fig. 3. Beyond 30 minutes of ischemia, Pi levels continued to rise, reaching $69.5 \pm 3.0 \%$ by the final (60-minute) acquisition period ( $p<0.05$ versus initial 30 minutes of ischemia). With this increase in $\mathrm{Pi}$, concurrent decreases were seen in levels of $\mathrm{PCr}$ (Fig. 4) and ATP (Fig. 5), While the level of PCr remained as low as the initial loss throughout the ischemic period, the decline of ATP appeared gradual and continuous, decreasing from $11.4 \pm 1.9 \%$ in the first 10 minutes to $4.8 \pm 0.7 \%$ at 60 minutes of ischemia.

With reperfusion, some similarities as well as differences between groups were observed. Animals in group 1 exhibited decreases in $\mathrm{Pi}$ levels, which fell to baseline values by the final reperfusion period. Group 2 animals showed a gradual decline in Pi levels after reperfusion, but by the final acquisition period, these levels remained significantly elevated above baseline values $(52.8 \pm 4.6 \%$ versus $22.1 \pm 2.0 \% ; p<0.05)$. Throughout the entire reperfusion period, group 1 rabbits had significantly lower levels of $P i$ than group 2 rabbits $(p<0.05)$.

Recovery of $\mathrm{PCr}$ stores also progressed differently in the two groups. Upon reperfusion, $\mathrm{PCr}$ in group 1 animals returned to baseline levels within the first spectral acquisition period, while group 2 animals did not regain pre-ischemic levels of $\mathrm{PCr}$ until the final period of reperfusion ( $p<0.05$ : group 1 versus group 2 for the second period of reperfusion). In both cases, the final level of $\mathrm{PCr}$ was significantly higher than that of early ( 0 to 20 minutes) occlusion $(p<0.05)$.

In terms of ATP levels, group 1 animals had a better recovery than did group 2 animals. For group 1, ATP levels approached baseline by 30 minutes of reperfusion ( $p=\mathrm{ns}$ versus baseline). However, in animals that underwent 60 minutes of ischemia, ATP levels remained markedly decreased compared with baseline $(p<0.05)$. Reperfusion after 60 minutes of ischemia halted further depletion of ATP in group 2, but after 30 minutes of reperfusion, significantly lower levels of ATP were detected in group 2 animals than in group 1 animals $(5.6 \pm 1.1 \%$ versus 10.8 $\pm 1.7 \% ; p<0.05)$.

Infarct size data (Table II). While no differences were present in the average weights of the left ventricle (2.7 versus $3.0 \mathrm{gm} ; p=\mathrm{ns})$ or risk area ( 1.3 versus $1.5 \mathrm{gm})$ or of the percentage at risk $(50 \%)$ between the two groups of animals, the mean weight of infarct $(0.4 \pm 0.2$ versus $0.9 \pm 0.3 \mathrm{gm}$, group 1 versus group 2 , respectively; $p<0.001$ ) along with the extent of viability was found to differ significantly. In group $1,86.8 \pm 7.7 \%$ of the left ventricle was viable tissue, whereas group 2 was found to have 


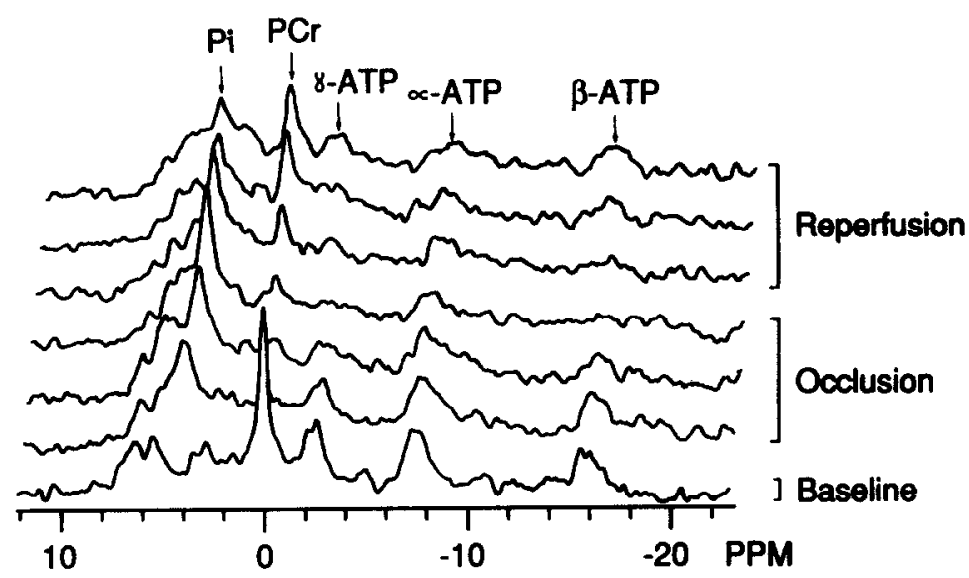

Fig. 1. Stacked plot of NMR data over time for group 1. One baseline, three ischemic, and three reperfusion spectra correspond to the time points outlined in Table I.

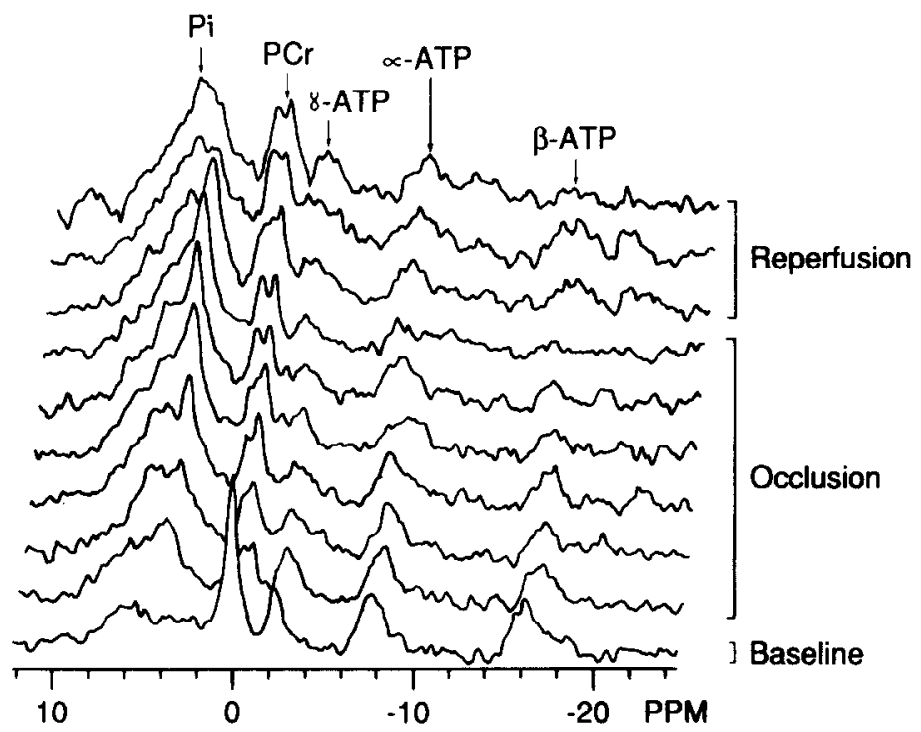

Fig. 2. Stacked plot of NMR data over time for group 2. One baseline, six ischemic, and three reperfusion spectra correspond to the time points outlined in Table $I$.

$69.6 \pm 8.1 \%$ of tissue remaining viable $(p<0.0005)$. The difference was more dramatic when the viability was expressed as a percentage of the risk area $(77.1 \pm 9.7 \%$ versus $39.4 \pm 9.4 \%, p<0.0001)$.

Correlations (Figs. 6 to 8). To evaluate correlations between myocardial viability and high-energy phosphate levels, several linear regression calculations were performed. Some reliable indicators of myocardial viability in this model were identified. During early reperfusion (within 10 minutes of ligature removal), both $\mathrm{Pi}$ and $\mathrm{PCr}$ levels correlated well with tissue salvage $(r=-0.65, p<0.01 ;$ and $r=54$, $p<0.05$, respectively). During the second period of reperfusion, all areas of phosphate peaks were shown to correlate well with myocardial viability (Pi:r = $-0.68, p<0.01$; PCr: $r=0.59, p<0.05$; ATP: $r=0.52$, $p<0.05$ ). Again, during the final accumulation of reperfusion data (30 minutes post-ischemia), $\mathrm{Pi}$ and PCr showed a significant correlation with the extent of viability $(r=-0.84, p<0.001$; and $r=53, p<0.05$, respectively). Averages taken over the entire 30minute reperfusion period indicate that both $\mathrm{Pi}$ and $\mathrm{PCr}$ were significant markers of myocardial viability in this experimental model $(r=-0.73, p<0.01$; and $r=59, p<0.05$, respectively). Expressed as the ratio of relative percent areas, $\mathrm{PCr} / \mathrm{Pi}$ correlated significantly with myocardial viability as well $(r=0.71$; $p<0.01$ ).

\section{DISCUSSION}

Our results indicate that high-energy phosphate metabolism measured by ${ }^{31} \mathrm{P}$ NMR may be useful in 
Table I. High-energy phosphate data

\begin{tabular}{|c|c|c|c|c|c|c|c|}
\hline \multirow[b]{2}{*}{ Time } & & \multicolumn{2}{|c|}{$P i$} & \multicolumn{2}{|c|}{$P C r$} & \multicolumn{2}{|c|}{$A T P$} \\
\hline & & Group I & Group 2 & Group 1 & Group 2 & Group 1 & Group 2 \\
\hline 0 & Baseline & $25.8 \pm 3.8$ & $22.1 \pm 2.0$ & $23.2 \pm 1.6$ & $23.9 \pm 1.8$ & $13.4 \pm 1.5$ & $16.3 \pm 2.0$ \\
\hline 10 & Occlusion & $44.9 \pm 5.5^{*}$ & $42.4 \pm 3.1^{*}$ & $12.4 \pm 2.9^{*}$ & $11.5 \pm 1.8^{*}$ & $9.2 \pm 1.3$ & $11.4 \pm 1.9^{*}$ \\
\hline 20 & & $46.7 \pm 4.2^{*}$ & $45.0 \pm 3.1^{*}$ & $12.2 \pm 2.1^{*}$ & $10.7 \pm 2.3^{*}$ & $9.5 \pm 12$ & $12.8 \pm 2.0$ \\
\hline 30 & & $46.4 \pm 4.0^{*}$ & $54.3 \pm 4.5^{*}$ & $16.7 \pm 2.5$ & $11.7 \pm 2.5^{*}$ & $7.1 \pm 1.5^{*}$ & $6.7 \pm 1.5^{*}$ \\
\hline 40 & & & $58.7 \pm 4.3^{*}$ & & $12.6 \pm 2.2^{*}$ & & $5.0 \pm 1.0^{*}$ \\
\hline 50 & & & $61.3 \pm 5.2^{*}$ & & $11.3 \pm 2.6^{*}$ & & $7.6 \pm 1.2^{*}$ \\
\hline 60 & & & $69.5 \pm 3.0^{*}$ & & $10.6+2.6^{*}$ & & $4.8+0.7^{*}$ \\
\hline 10 & Reperfusion & $41.6 \pm 4.4^{*}$ & $59.4 \pm 6.4^{* \dagger}$ & $24.3 \pm 2.7$ & $16.5 \pm 3.9^{*}$ & $7.4 \pm 1.4^{*}$ & $5.2 \pm 1.2^{*}$ \\
\hline 20 & & $38.4 \pm 4.5^{*}$ & $57.1 \pm 5.7^{*} \dagger$ & $24.8 \pm 2.9$ & $16.1 \pm 2.7^{*} \dagger$ & $8.7 \pm 1.5^{*}$ & $5.8 \pm 1.0^{*}$ \\
\hline 30 & & $35.4 \pm 4.0$ & $5.28 \pm 4.6^{* \dagger}$ & $23.8 \pm 3.1$ & $21.9 \pm 1.8$ & $10.8 \pm 1.7$ & $5.6 \pm 1.1^{*} \dagger$ \\
\hline
\end{tabular}

NMR data expressed as peak area/total area of spectrum $\times 100$. Means \pm SEM. Group $1=30$ minutes of ischemia. Group $2=60$ minutes of ischemia. $\mathrm{Pi}$, Inorganic phosphate; $\mathrm{PCr}$, phosphocreatine; ATP, adenosine triphosphate.

${ }^{*} p<0.05$ versus baseline; $\dagger p<0.05$ between groups.

Table II. Viability and infarct size data

\begin{tabular}{cccc}
\hline & \multicolumn{3}{c}{ LV weights $(\mathrm{gm})$} \\
\cline { 2 - 4 } Group & Total & Risk & Infarct \\
\hline 1 & $2.7 \pm 0.2$ & $1.3 \pm 0.1$ & $0.4 \pm 0.1$ \\
2 & $3.0 \pm 0.2$ & $1.5 \pm 0.1$ & $0.9 \pm 0.1^{*}$ \\
\hline
\end{tabular}

Ratios

\begin{tabular}{ccclll} 
& Risk/LV & Viable/LV & Viable/risk area & Infarct/LV & Infarct/risk \\
\hline 1 & $50 \pm 6$ & $86.8 \pm 2.7$ & $77.1 \pm 4.1$ & $13.0 \pm 2.7$ & $23.0 \pm 4.1$ \\
2 & $50 \pm 5$ & $69.6 \pm 2.7^{*}$ & $39.4 \pm 3.4^{*}$ & $30.2 \pm 2.7^{*}$ & $60.6 \pm 3.4^{*}$ \\
\hline
\end{tabular}

All data expressed as mean \pm SEM.

Group $1=30$ minutes of ischemia. Group $2=60$ minutes of ischemia.

$L V$, Left ventricle.

${ }^{*} p<0.001$ versus group 1 .

directly evaluating myocardial viability of the left ventricle following coronary occlusion and reperfusion. The differences in HEP recovery observed between the two study groups indicate the metabolic effects following different durations of ischemia. The inability of severely injured myocardium to restore levels of ATP while retaining high levels of $\mathrm{Pi}$ suggests the importance of the extent of salvaged myocardial tissue in the maintenance of HEP metabolic integrity, which ultimately determines the potential for myocardial contractility. Moreover, the significant correlations of tissue viability with the levels of $\mathrm{PCr}$ present during myocardial reperfusion as well as its inverse relationship to inorganic phosphates suggest the possibility of utilizing these parameters to assess salvaged myocardial tissue. Clearly, the ability to determine the extent of tissue that has been salvaged following interventions such as coronary reperfusion has tremendous clinical relevance. Furthermore, the ${ }^{31} \mathrm{P}$ NMR spectroscopic assessment of HEP metabolism as an indication of tissue viability may have significant advantages over other available techniques.

A wide variety of other methods have been used in an attempt to assess myocardial viability following coronary reperfusion. However, many of these techniques have major limitations that restrict their ability to accurately evaluate the viability of myocardial tissue. For example, thallium 201 scintigraphy is limited by complex ${ }^{201} \mathrm{Tl}$ kinetics, which may be markedly altered around the time of reperfusion. ${ }^{16,17}$ Furthermore, ${ }^{201} \mathrm{Tl}$ can accumulate in necrotic as well as in viable tissue, resulting in an overestimation of tissue salvage. ${ }^{2}$ Functional assessment, including contrast and radionuclide angiography and two-dimensional echocardiography, may in- 


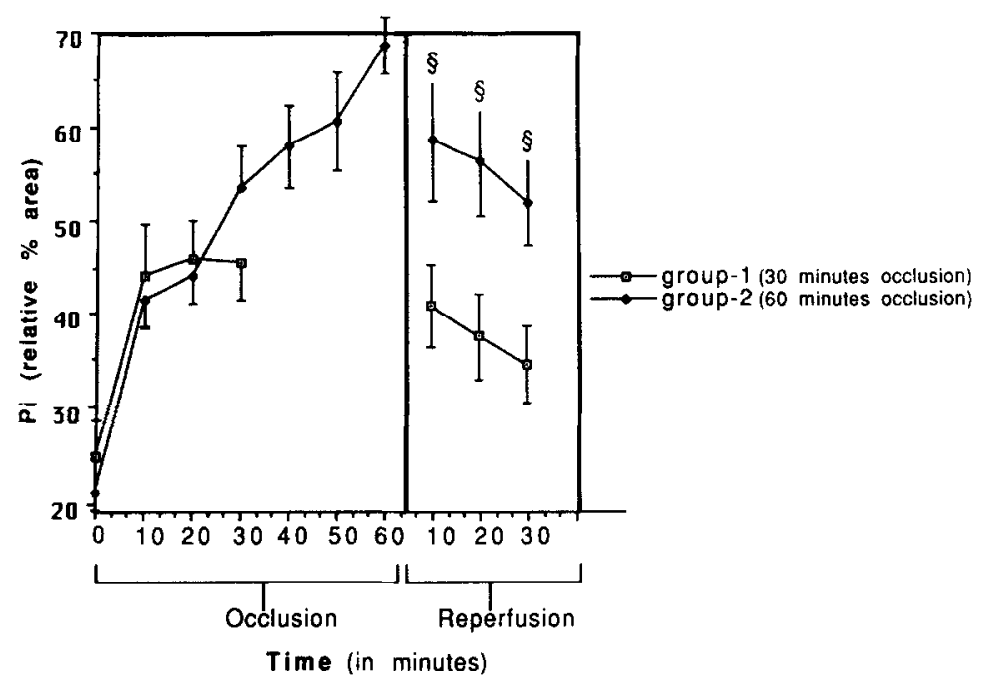

Fig. 3. Changes in inorganic phosphates $(P i)$ over time. The graph is divided into occlusion and reperfusion periods. Group 1 animals do not have values for the ischemic period 40 to 60 minutes. $\$ p<0.05$ between groups.

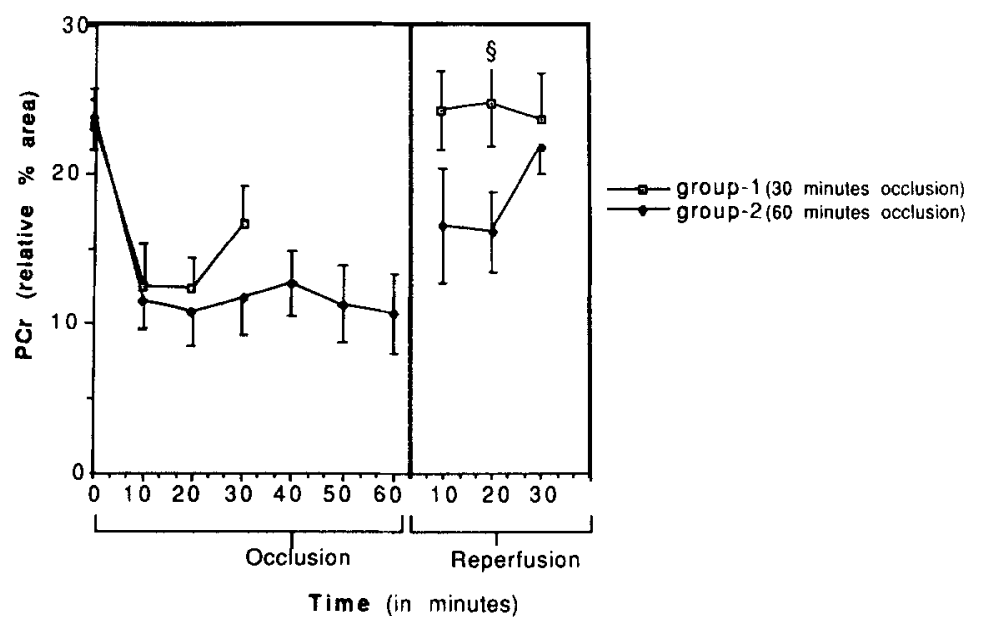

Fig. 4. Changes in phosphocreatine $(P C r)$ over time. The graph is divided into occlusion and reperfusion periods. Group 1 animals do not have values for the ischemic period 40 to 60 minutes. $\S p<0.05$ between groups.

accurately characterize viable tissue as irreversibly damaged due to the post-reperfusion phenomenon of temporarily stunned myocardium. ${ }^{1}$ Especially when utilized early after reperfusion, these techniques are of limited usefulness and may significantly underestimate the extent of tissue viability.

More recently, positron emission tomography has emerged as the preferred technique for assessment of tissue salvage. The uptake and clearance of ${ }^{11} \mathrm{C}$ palmitic acid indicates the extent of fatty acid metabolism, but this process of energy production is compromised in oxygen-deprived ischemic myocardium. ${ }^{3}$ Using ${ }^{18} \mathrm{~F}$-fluorodeoxyglucose (FDG) and lactate production, the anaerobic glycolytic pathway may be followed, indicating the capacity for substrate utilization within the myocardium and consequently its viability. The continuation of anaerobic metabolism, while inferior to the highly energy-productive oxidative phosphorylation, indicates that myocytes, although damaged, remain viable. ${ }^{19}$ Because blood flow and FDG uptake are reduced in infarcted tisue, ${ }^{20}$ characterization of tissue through FDG uptake or glucose utilization ${ }^{21}$ may be possible. However, reported relationships between FDG uptake and the extent of necrosis ${ }^{3}$ are not superior to the correlations between HEP levels and viability reported here, using ${ }^{31} \mathrm{P}$ NMR. The evaluation of high-energy phosphate stores through ${ }^{31} \mathrm{P}$ NMR may provide the most direct information about the viability and contractile potential of reperfused myocardium and may further 


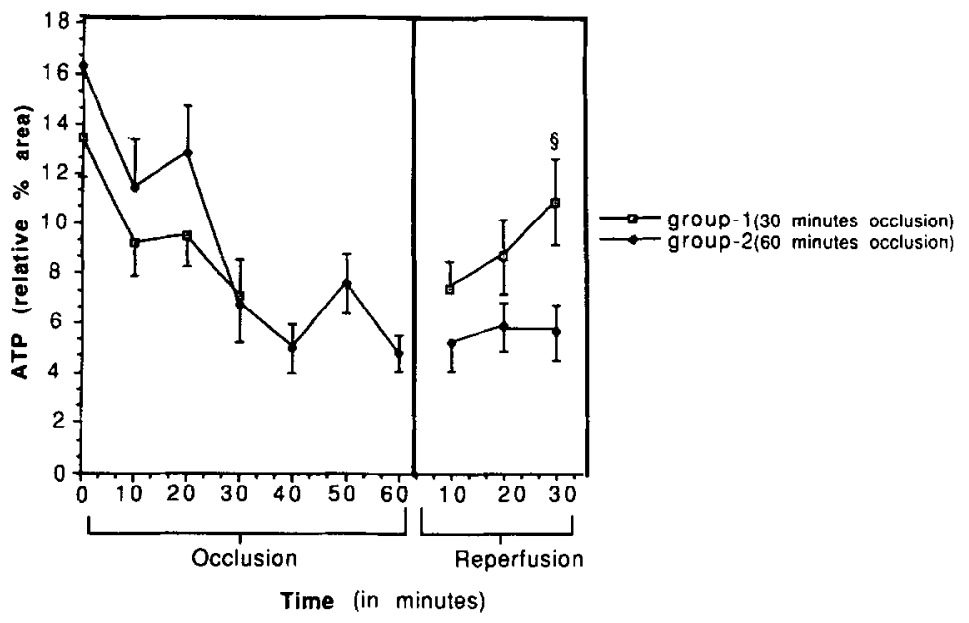

Fig. 5. Changes in ATP over time. The graph is divided into occlusion and reperfusion periods. Group 1 animals do not have values for the ischemic period 40 to 60 minutes. $\S p<0.05$ between groups.

complement the metabolic information assessed by positron tomography.

Rehr et al. ${ }^{13}$ have recently investigated the potential of ${ }^{31} \mathrm{P}$ NMR spectroscopy to characterize myocardial tissue. They examined two experimental groups of dogs wherein one group underwent permanent sustained occlusion while the other underwent 1 hour of occlusion followed by reperfusion. The resulting infarct sizes, expressed as a percentage of the risk region, were widely disparate $(14 \pm 8 \%$ versus $109 \pm 11 \% ; p<0.0001)$. They suggested that the analysis of seven metabolic variables $(\mathrm{pH}, \mathrm{PCr}, \mathrm{ATP}$, $\mathrm{Pi}$, and the ratios of $\mathrm{PCr} / \mathrm{ATP}, \mathrm{Pi} / \mathrm{PCr}, \mathrm{Pi} / \mathrm{ATP}$ ) may provide sufficient information about the condition of myocardial tissue to determine its viability. In their study, they were able to distinguish normal, ischemic, and reperfused yet viable myocardium by logistic regression analysis with an overall accuracy of $89 \%$. In a subsequent study by the same group of investigators, ${ }^{22}$ the accuracy of using the ${ }^{31} \mathrm{P}$ NMR data for distinguishing viable from infarcted myocardium was $100 \%$, and their results indicated that PCr was the metabolic variable permitting most effective separation of viable and infarcted tissue. Their results, in concert with our own data, support the hypothesis that HEP metabolism, as evaluated by NMR spectroscopy, may be valuable in the characterization of myocardial tissue viability.

The different durations of occlusion utilized in our investigation produced varying degrees of myoardial injury and various degrees of metabolic recovery following coronary reperfusion. Animals were subjected to either 30 or 60 minutes of ischemia, as these time intervals have been described as distinctly different in the development of necrotic tissue in rabbits. ${ }^{23} \mathrm{In}$

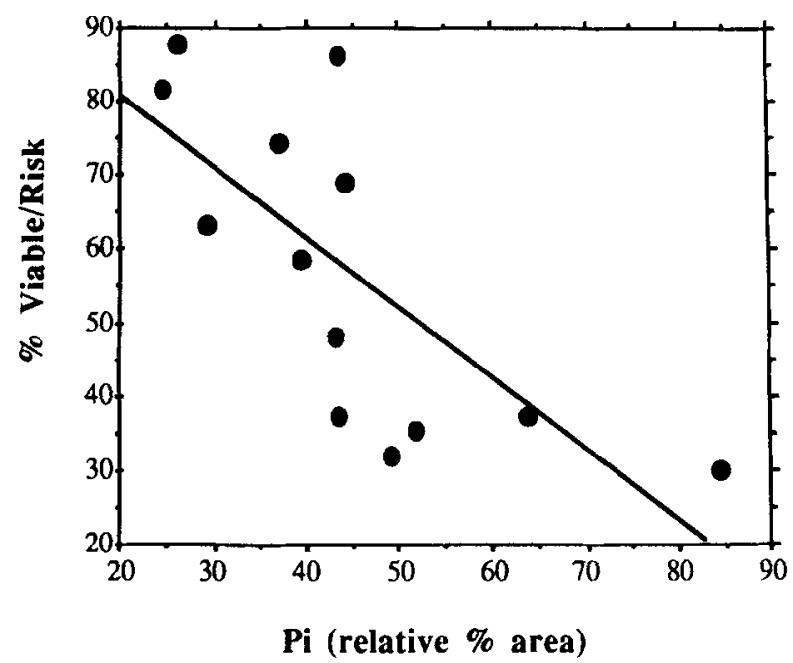

Fig. 6. Linear regression plot representing the correlation between the percent of viable myocardium and the level of inorganic phosphates during the reperfusion period. $r=0.73 ; \mathrm{y}=0.543 \mathrm{x}+65.596 ; p<0.01$.

the intact rabbit, 15 to 30 minutes of ischemia results in significantly less left ventricular necrosis than 60 minutes of ischemia, which often causes entire transmural infarcts. The differences in metabolic recovery observed following the two periods of ischemic injury suggest a continuum of metabolic recovery following reperfusion reflecting the extent of salavaged viable myocardium.

Recently, Wolfe et al. ${ }^{24}$ examined ${ }^{31} \mathrm{P}$ NMR spectra in a rodent model of reversible myocardial ischemia using a modified solenoid coil implanted around the heart. In agreement with our results, animals subjected to 30 minutes of myocardial ischemia 


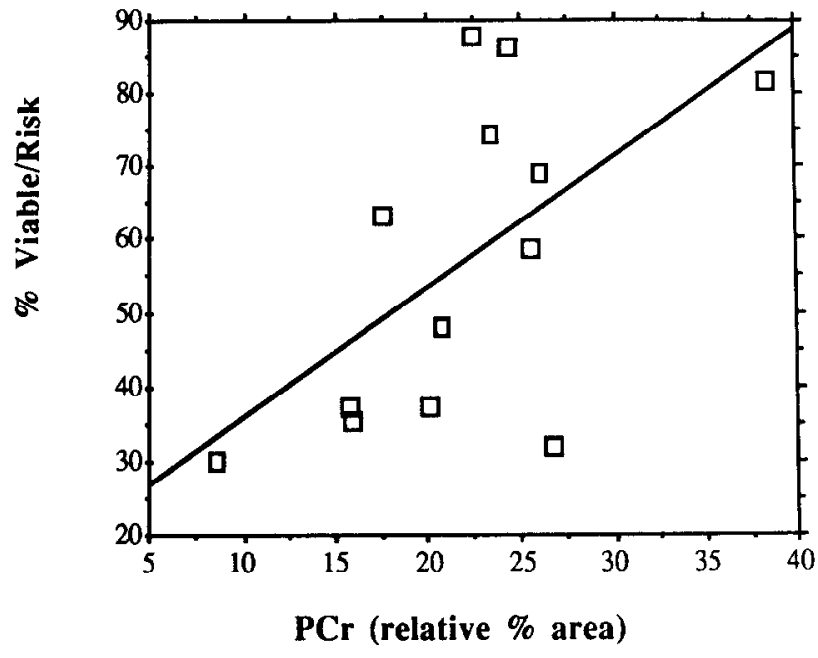

Fig. 7. Linear regression plot representing the correlation between the percent of viable myocardium and level of phosphocreatine during reperfusion period. $r=0.59$; $\mathrm{y}=0.194 \mathrm{x}+10.891 ; p<0.05$.

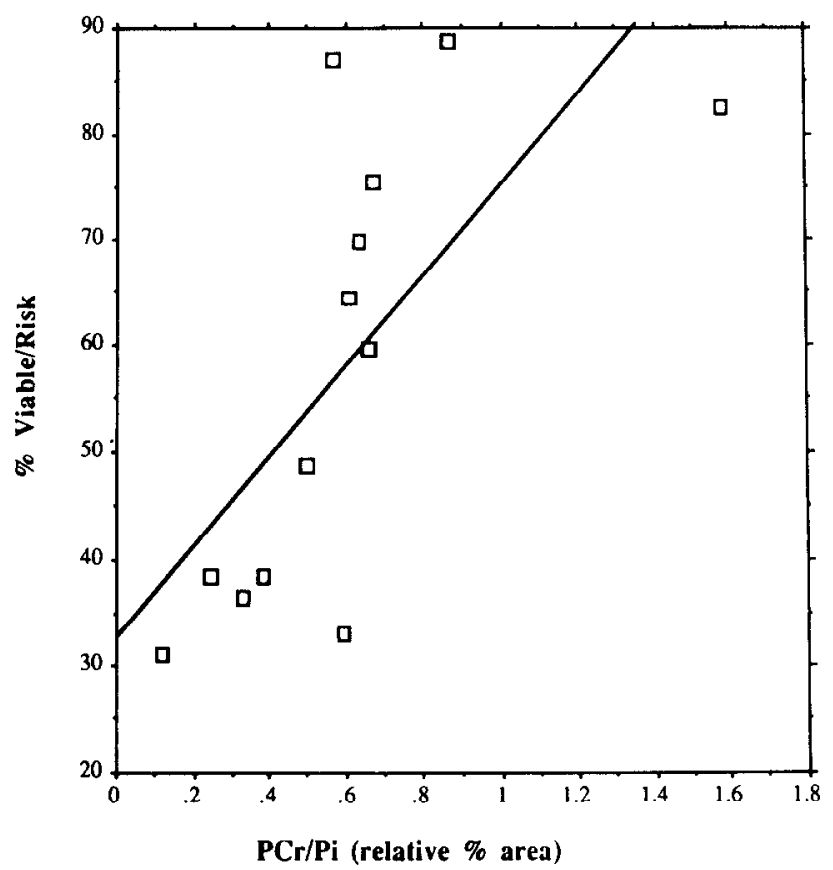

Fig. 8. Linear regression plot representing the correlation between the percent of viable myocardium and the ratio of the relative percent areas of $\mathrm{PCr} / \mathrm{Pi} . r=0.71 ; \mathrm{y}=42.1 \mathrm{x}$ $+31.9 ; p<0.01$.

showed significant $\mathrm{PCr}$ recovery, whereas those subjected to 60 minutes of ischemia had no $\mathrm{PCr}$ recovery. Also in accord with our results, $\beta$-ATP was reduced during ischemia and did not recover in the initial reperfusion period. However, these investigators did not perform quantitative analysis of risk re- gion or infarct size, and did not report correlations between metabolic results and salvaged, viable myocardium. In this regard, our data further extend their observations by demonstrating that a significant correlation exists between the degree of metabolic recovery and the tissue viability of salvaged myocardium.

To our knowledge, the only other study to investigate a wide range of myocardial injury in the rabbit using ${ }^{31} \mathbf{P}$ NMR utilized the isolated heart preparation. ${ }^{25}$ Periods of ischemia ranging from 10 to 60 minutes produced changes in HEP metabolism and function recovery. The in vitro study, which accumulated data with a $4.7 \mathrm{~T}$ magnet, reported end-ischemic ATP levels as the best predictor of left ventricle functional recovery. The failure of our study to identify ATP as a reliable predictor of myocardial viability is in accordance with the findings of other reports of metabolic recovery in reperfused myocardium. ${ }^{4,13,26,27}$ Ellis et al. ${ }^{28}$ have demonstrated that following 90 minutes of coronary occlusion, intact dogs did not recover baseline levels of ATP until 7 days after reperfusion. The limited production of ATP through anaerobic glycolysis, which may persist even after coronary reperfusion has occurred, ${ }^{29,30}$ the slow synthesis of adenine nucleotides through the de novo or salvage pathways, ${ }^{4}$ or the poor spectral resolution at the frequency of $\beta$-ATP, may account for the inability of post-reperfusion ATP levels to predict tissue viability in our model.

Our results suggest that ${ }^{31} \mathrm{P}$ NMR may further complement the current evaluations of myocardial tissue viability by assessing the status of high-energy phosphate metabolism, which directly indicates the extent of tissue salvage. Furthermore, there are several advantages of ${ }^{31} \mathrm{P}$ NMR spectroscopy over PET imaging and other methods of viability assessment. The most obvious is that ${ }^{31} \mathrm{P}$ NMR spectroscopy is potentially totally noninvasive with the use of a surface coil. This would allow serial evaluation of the post-infarct patient to determine HEP metabolic capacity. Thus alterations in the extent of tissue viability over time could be examined, or alternatively, alterations of viability following various myocardial interventions could be assessed. Using ${ }^{31} \mathrm{P}$ NMR, specific biochemical processes can be quantified, such as the flux of the creatine kinase reaction vital to ATP synthesis through saturation transfer kinetic investigations, an "invisible labeling" technique. ${ }^{31}$ However, ${ }^{31} \mathrm{P}$ NMR requires further technical improvements, including the development of a three-dimensional volume localization process, compensation for saturation effects, and improvement in instrumentation to decrease the signal-to-noise ratio, 
before this technique can be reliably applied to humans.

The assistance in data analysis and computer processing provided by Kevin Kavanaugh, MD, is greatly appreciated by the authors.

\section{REFERENCES}

1. Braunwald E, Kloner RA. The stunned myocardium: prolonged, postischemic ventricular dysfunction. Circulation 1982;66:1146-9.

2. Melin JA, Becker LC, Bulkley BH. Differences in thallium-201 uptake in reperfused and nonreperfused myocardial infarction. Circ Res 1983;53:414-9.

3. Schwaiger M, Schelbert HR, Ellison D, Hansen H, Yeatman L, Vinten-Johansen J, Selin C, Barrio J, Phelps ME. Sustained regional abnormalities in cardiac metabolism after transient ischemia in the chronic dog model. J Am Coll Cardiol 1985; 6:336-47.

4. Reimer KA, Hill ML, Jennings RB. Prolonged depletion of ATP and of the adenine nucleotide pool due to delayed resynthesis of adenine nucleotides fullowing reversible myocardial ischemic injury in dogs. J Mol Cell Cardiol 1981;13:229-39.

5. Kavanaugh KM, Aisen AM, Fechner KP, Chenevert T, Dunham WR, Buda AJ. Regional metabolism during coronary occlusion, reperfusion, and reocclusion using phosphorus-31 nuclear magnetic resonance spectroscopy in the intact rabbit. $\mathrm{AM}$ HEART J 1989;117:53-9.

6. Guth BD, Martin JF, Heusch G, Ross J. Regional myocardial blood flow, function and metabolism using phosphorous-31 nuclear magnetic resonance spectroscopy during ischemia and reperfusion in dogs. J Am Coll Cardiol 1987;10:673-81.

7. Brown JJ, Strich G, Higgins CB, Gerber KH, Slutsky RA. Nuclear magnetic resonance analysis of acute myocardial infarction in dogs: the effects of transient coronary ischemia of varying duration and reperfusion on spin lattice relaxation times. AM HEART J 1985;109:486-90.

8. Lavanchy N, Martin J, Rossi A. Graded global ischaemia and reperfusion of the isolated perfused rat heart: characterisation by 31-P NMR spectroscopy of the extent of energy metabolism damage. Cardiovasc Res 1984;18:573-82.

9. Schaefer S, Gober J, Valenza M, Karczmar GS, Matson GB, Camacho SA, Botvinick EH, Massie B, Weiner MW. Nuclear magetic resonance imaging-guided phosphorus-31 spectroscopy of the human heart. J Am Coll Cardiol 1988;12:1449-55.

10. Bottomley PA, Herfkens RJ. Smith LS, Brazzamano S, Blinder R, Hedlund LW, Swain JL, Redington RW. Noninvasive detection and monitoring of regional myocardial ischemia in situ using depth-resolved 31-P NMR spectroscopy. Proc Natl Acad Sci 1985;82:8747-51.

11. Blackledge MJ, Rajagopalan B, Oberhaensli RD, Bolas NM, Styles P, Radda G. Quantitative studies of human cardiac metabolism by 31-P rotating-frame NMR. Proc Natl Acad Sci USA 1987;84:4283-7.

12. Whitman GR, Chance B, Bode H. Diagnosis and therapeutic evaluation of pediatric cardiomyopathy using 31-P NMR. J Am Coll Cardiol 1985;5:745-9.

13. Rehr RB, Tatum JL, Hirsch JI, Wetstein L, Clarke G. Effective separation of normal, acutely ischemic, and reperfused myocardium with P-31 MR spectroscopy. Radiology 1988; 168:81-9.

14. Murphy-Boesch J, Koretsky AP. An in vivo NMR probe circuit for improved sensitivity. J Magn Reson 1983;54:526-32.

15. Fishbein MC, Meerbaum S, Rit J, Lando U, Kanmatsuse K, Mercier JC, Corday E, Ganz W. Early phase acute myocardial infarct size quantification: validation of the triphenyl tetrazolium chloride tissue enzyme staining technique. AM HEART J 1981;101:593-600.
16. Forman R, Kirk ES. Thallium-201 accumulation during reperfusion of ischemic myocardium: dependence on regional blood flow rather than viability. Am J Cardiol 1984;54:659-63.

17. Okada RD, Pohost GM. The use of pre-intervention and postintervention thallium imaging for assessing the early and late effects of experimental coronary arterial reperfusion in dogs. Circulation 1984;69:1153-60.

18. Schelbert HR, Henze E, Schon HR, Keen R, Hansen H, Selin C, Huang SC, Barrio JR, Phelps ME. C-11 palmitate for the noninvasive evaluation of regional myocardial fatty acid metabolism with positron computed tomography. III. In vivo demonstration of the effects of substrate availability on myocardial metabolism. AM HEART J 1983;105:492-504.

19. Jennings RB, Reimer KA, Hill ML, Mayer SE. Total ischemia in dog hearts, in vitro. I. Comparison of high energy phosphate production, utilization, and depletion, and of adenine nucleotide catabolism in total ischemia in vitro vs. severe ischemia in vivo. Circ Res 1981;49:892-900.

20. Marshall RC, Tillisch JH, Phelps ME, Huang SC, Carson R, Henze E, Schelbert HR. Identification and differentiation of resting myocardial ischemia and infarction in man with positron computed tomography, ${ }^{18} \mathrm{~F}$-labeled fluorodeoxyglucose and N-13 ammonia. Circulation 1983;67:766-77.

21. Schwaiger M, Neese RA, Araujo L, Wyns W, Wisneski JA, Sochor H, Swank S, Kulber D, Selin C, Phelps M, Schelbert H, Fishbein MC, Gertz EW. Sustained nonoxidative glucose utilization and depletion of glycogen in reperfused canine myocardium. J Am Coll Cardiol 1989;13:745-54.

22. Rehr RB, Tatum JL, Hirsch JI, Quint R, Clarke G. Reperfused-viable and reperfused-infarcted myocardium: differentiation with in-vivo P.31 NMR spectroscopy. Radiology 1989;172:53-8.

23. Connelly C, Vogel VM, Hernandez YM, Apstein CS. Movement of necrotic wavefront after coronary artery occlusion in rabbit. Am J Physiol 1982;243:H682-90.

24. Wolfe CL, Moseley ME, Wikstrom MG, Sievers RE, Wendlund MF, Dupon JW, Finkbeiner WE, Lipton MJ, Parmley WW, Brasch RC. Assessment of myocardial salvage after ischemia and reperfusion using magnetic resonance imaging and spectroscopy. Circulation 1989;80:969-82.

25. Whitman GJR, Kieval RS, Seeholzer S, McDonald G, Simson MB, Harken AH. Recovery of left ventricular function after graded cardiac ischemia as predicted by myocardial P-31 nuclear magnetic resonance. Surgery 1985;97:428-34.

26. Murphy ML, Peng CF, Kane JJ Jr, Straub KD. Ventricular performance and biochemical alteration of regional ischemic myocardium after reperfusion in the pig. Am $\mathrm{J}$ Cardiol 1982;50:821-8.

27. Peng CF, Davis JL, Murphy ML, Straub KD. Effects of reperfusion on myocardial wall thickness, oxidative phosphorylation, and $\mathrm{Ca}^{2+}$ metabolism following total and partial myocardial ischemia. AM HEART J 1986;112:1238-44.

28. Ellis SG, Henschke CI, Sandor T, Wynne J, Braunwald E, Kloner RA. Time course of functional and biochemical recovery of myocardium salvaged by reperfusion. J Am Coll Cardiol 1983;1:1047-55

29. Schwaiger M, Schelbert HR, Keen R, Vinten-Johansen J, Hansen H, Selin C, Barrio J, Huang SC, Phelps ME. Retention and clearance of $\mathrm{C}-11$ palmitic acid in ischemic and reperfused canine myocardium. J Am Coll Cardiol 1985;6:31120.

30. Brown MA, Myears DW, Herrero P, Bergmann SR. Disparity between oxidative and fatty acid metabolism in reperfused myocardium assessed with positron emission tomography [Abstract]. Circulation 1987;76(suppl IV):IV-4.

31. Neubauer S, Hamman BL, Perry SB, Bittl JA, Ingwall JS. Velocity of the creatine kinase reaction decreases in postischemic myocardium: a \$1-P.NMR magnetization transfer study of the isolated ferret heart. Circ Res 1988;63:1-15. 